\title{
Preliminary clinical application of foldable capsular vitreous body in severe silicone oil-dependent eyes
}

\author{
$\mathrm{Na}$ Liu ${ }^{1,2 \#}$, Ling Kang ${ }^{1,2 \#}$, Xi Yu ${ }^{1,2}$, Qingqing $\mathrm{Lv}^{1,2}$, Lei $\mathrm{Xi}^{1,2}$, Meifang Liu $^{1,2}$, Yong Liu ${ }^{1,2}$, Nan Wu ${ }^{1,2}$ \\ ${ }^{1}$ Department of Ophthalmology, Southwest Hospital/Southwest Eye Hospital, Third Military Medical University (Army Medical University), \\ Chongqing, China; 'Key Laboratory of Visual Damage and Regeneration and Restoration of Chongqing, Chongqing, China \\ Contributions: (I) Conception and design: N Wu, N Liu; (II) Administrative support: N Wu, Y Liu; (III) Provision of study materials or patients: \\ N Wu, N Liu, X Yu; (IV) Collection and assembly of data: N Liu, L Kang, X Yu; (V) Data analysis and interpretation: N Liu, L Kang, Q Lv; (VI) \\ Manuscript writing: All authors; (VII) Final approval of manuscript: All authors. \\ "These authors contributed equally to this work. \\ Correspondence to: Nan Wu. Department of Ophthalmology, Southwest Hospital/Southwest Eye Hospital, Third Military Medical University (Army \\ Medical University), 30 Gaotanyan Street, Shapingba District, Chongqing 400038, China. Email: nanwu1122@163.com.
}

\begin{abstract}
Background To investigate the clinical safety and efficacy of foldable capsular vitreous body (FCVB) in the treatment of severe silicone oil-dependent eyes.

Methods: Five patients ( 5 eyes) of severe silicone oil-dependent eyes underwent FCVB implantation from December 2019 to July 2020. The preoperative and postoperative visual acuity, intraocular pressure, corneal conditions, postoperative retinal reattachment, and FCVB status were observed. The patients were followed up for 12-15 months.
\end{abstract}

Results: A total of 5 patients (5 eyes) with severe silicone oil-dependent eyes were included, including 3 males and 2 females, with an average age of $32 \pm 14$ years. The longest silicone oil tamponade time was 8 years, and the shortest was 2 years, with an average of $5 \pm 3$ years. All patients were safely and successfully implanted with FCVB without severe intraoperative or postoperative complications. Up to the last followup, there was no significant change between preoperative and postoperative visual acuity. Before surgery, 4 patients had normal intraocular pressure while 1 patient had ocular hypotension; after surgery, the latter was still at a low level, and the other patients had intraocular pressure in the normal range.

Conclusions: The FCVB is a safe, feasible, and effective vitreous substitute to maintain ocular morphology and intraocular pressure without aggravating silicone oil emulsification, retinal displacement, or corneal degeneration during the implantation period of 12-15 months.

Keywords: Silicone oil-dependent eyes; foldable capsular vitreous body (FCVB); vitrectomy

Submitted Aug 19, 2021. Accepted for publication Oct 16, 2021.

doi: 10.21037/apm-21-2554

View this article at: https://dx.doi.org/10.21037/apm-21-2554

\section{Introduction}

Vitrectomy is always required for treatment of vitreoretinal disease or eye injuries. Because the natural vitreous body cannot regenerate, vitreous substitutes need to be filled into the eyes to support retinal repairment $(1,2)$. Commonly used vitreous substitutes still have serious disadvantages such as short intraocular permanence, significant toxicity, and more complications (3). Silicone oil is widely used intraocular filling material during pars plana vitrectomy (PPV), some eyes were depended on silicone oil filling for severe ocular trauma or complex retinal detachment. Silicone oil removal may cause repeated retinal detachment, even eyeball atrophy, and the current vision was always poor or blind. There were lots of complications for silicon oil dependent; such as cataract, secondary glaucoma, corneal banded degeneration, silicone oil emulsification, and so on, multiple surgeries may require (4). The patients 
Table 1 Contraindications for FCVB implantation

\begin{tabular}{ll}
\hline Number & Contraindication \\
\hline 1 & Allergic to silica gel, scar diathesis \\
3 & Endophthalmitis \\
4 & Uveitis \\
5 & Transparent lens in the operated eye \\
6 & Proliferative diabetic retinopathy \\
7 & Contralateral corrected visual acuity $\leq 0.4$ \\
8 & History of intraocular surgery in contralateral eyes \\
9 & Other uncontrollable ocular comorbidities \\
10 & Severe renal and liver function damage and/or severe systemic diseases (such cardiovascular system, respiratory system, \\
11 & digestive system, nervous system, endocrine system, urogenital system diseases, etc.) \\
\hline
\end{tabular}

FCVB, foldable capsular vitreous body.

therefore require multiple surgeries or even enucleation. Foldable capsular vitreous body (FCVB) is an innovative intraocular tamponade product that can not only effectively support the eyeball, but also isolate the direct contact between silicone oil and the eye. The FCVB preserves the advantages of silicone oil while preventing complications such as silicone oil emulsification, secondary glaucoma, and corneal degeneration. FCVB is a vitreous substitute that can be implanted in the eye for a long time, and its safety and efficacy have been preliminarily confirmed by serial studies from animal experiments to standard development to clinical trials (5-8). As an artificial material that can save the eyeball, the implantation of FCVB can avoid various complications caused by silicone oil-dependent eyes or even enucleation, maintain the shape of the eyeball, and therefore improve patient quality of life. Additionally, this product can reduce the economic burden and mental and physical pain of patients. However, the clinical application of FCVB has not been widely promoted, and the number of cases in previous clinical studies are few. The main use of FCVB is eyebulb preservation, rather than restoration of vision, especially for the patients whose eyes have no visual improved potential, these eyes always are performed repeated operations, due to traumatic retinal detachment (9). Therefore, we retrospectively summarized the patients who underwent FCVB implantation in severe silicone oildependent eyes, to observe the complications of FCVB postoperatively, and demonstrate the safety and efficacy of FCVB in clinical application.

We present the following article in accordance with the STROBE reporting checklist (available at https://dx.doi. org/10.21037/apm-21-2554).

\section{Methods}

\section{General information}

Five patients underwent FCVB implantation for severe silicone oil-dependent eyes from December 2019 to July 2020 in the Department of Ophthalmology, the Southwest Hospital of Army Medical University. The clinical data were retrospectively analyzed. The exclusion criteria referred to the contraindications for FCVB (Table 1). Based on a strong desire for surgical treatment, all patients and their families were informed of the relevant information of surgery and implants in detail and signed the informed consent. This study was conducted in accordance with the Declaration of Helsinki (as revised in 2013) and was reviewed and approved by the Ethics Committee of the Southwest Hospital of Army Medical University (KY2021066). Informed consent was obtained from all the patients. All procedures were performed by the same physician. The time of follow-up was $12-15$ months, with an average of $13.5 \pm 1.5$ months. 


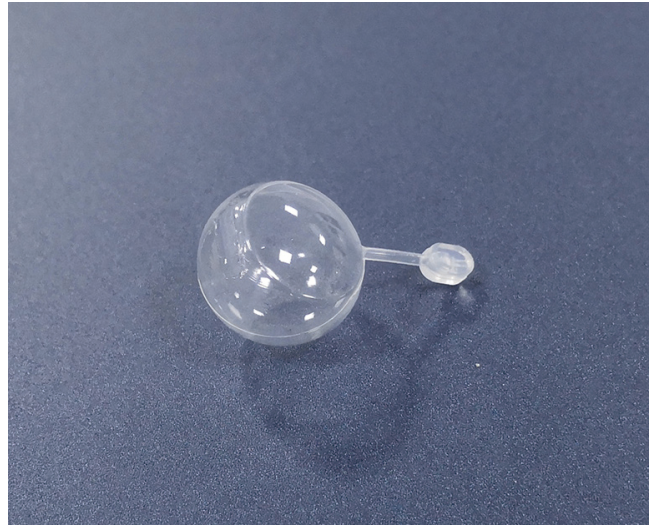

Figure 1 Profile display of FCVB. FCVB, foldable capsular vitreous body.

\section{Methods}

\section{Surgical procedures}

All patients underwent FCVB (Guangzhou Vesber Biotechnology, Guangzhou, China) implantation (Figure 1). Surgical procedures were performed as follows: (I) after successful intravenous general anesthesia, the surgical eye was routinely disinfected and draped. (II) A standard channel for $23 \mathrm{G}$ vitrectomy was made $3.5 \mathrm{~mm}$ posterior to corneal limbus in the superior temporal, inferior temporal, and superior nasal quadrants, and perfusion was connected inferotemporally. After aspiration of silicone oil, the retina was examined, and corresponding peeling and laser treatments were performed according to the retinal condition with air-fluid exchange. The visual retina was restored as flat as possible. (III) The airtightness of the FCVB was examined underwater. (IV) The FCVB was folded into the injector. (V) With the diameter of the superior scleral puncture site enlarged to $3.5-4.0 \mathrm{~mm}$, the injector head was advanced into the vitreous cavity. In case of detachment of ciliary body, FCVB was implanted from the enlarged contralateral incision. (VI) The lens part of the FCVB was upwards, and the FCVB was fully unfolded to support the retina. (VII) Silicone oil was slowly pushed in from the drainage valve of the FCVB. If the artificial vitreous body was tilted, the position could be appropriately adjusted with a blunt instrument to fill until the intraocular pressure almost reached normal by finger tension. (VIII) An optical fiber was inserted from the superior temporal puncture site, and retina condition and optic disc color were observed using ophthalmoscope. (IX) The FCVB drainage tube was ligated and fixed under the fornix conjunctiva which was then sutured.

\section{Perioperative medication}

Antibiotic eye drops were locally applied 1 day before surgery, 4 times a day, which was continued locally 1 day after surgery, 4 times a day. Systemic and local corticosteroids for anti-inflammation were given within 2 months after surgery.

\section{Observation methods}

The participants were followed up for 12-15 months. Their visual acuity, intraocular pressure, corneal endothelium (SP-3000P, Topcon, Tokyo, Japan), anterior chamber of the eyes, ocular fundus, and retinal reattachment were examined before surgery and at 1 week, 2 weeks, 1 month, 3 months, and 6 months after surgery. The observation and followup were completed through optical coherence tomography (OCT) (Spectralis, Heidelberg, Germany), ultrasound biomicroscopy (UBM) (SW-3200, Suowei, Tianjin, China), B ultrasound (Quantel Medical Cinescan, Cournond'Auvergne, France), color fundus photography (TRC50EX, Topcon, Japan), and anterior segment photography. Therefore, the success rate of surgery, stability of FCVB implantation, retinal reattachment, and intraoperative and postoperative complications were recorded.

\section{Results}

\section{General conditions}

There were 5 cases ( 5 eyes), including 3 males ( 3 eyes) and 2 females ( 2 eyes), aged 18-46 years (average age: $32 \pm 14$ years). Among them, there were 3 patients with ocular injury such as retinal re-detachment after surgery, and with silicone oil-dependent after multiple vitrectomies. The other 2 patients with retinal detachment and silicone oil-dependent after multiple vitrectomy combined with scleral buckling. The longest silicone oil filling time was 8 years, and the shortest was 2 years, with an average of $5 \pm 3$ years.

\section{Surgical safety and effectiveness}

The FCVB was successfully implanted in all 5 patients. No intraoperative complications such as choroidal hemorrhage, FCVB rupture, inability to inject silicone oil, or silicone oil spillage were recorded.

A single case developed hyphema 1 day after operation, which was absorbed and improved 2 weeks after hemostasis and other symptomatic treatment, without rebleeding 

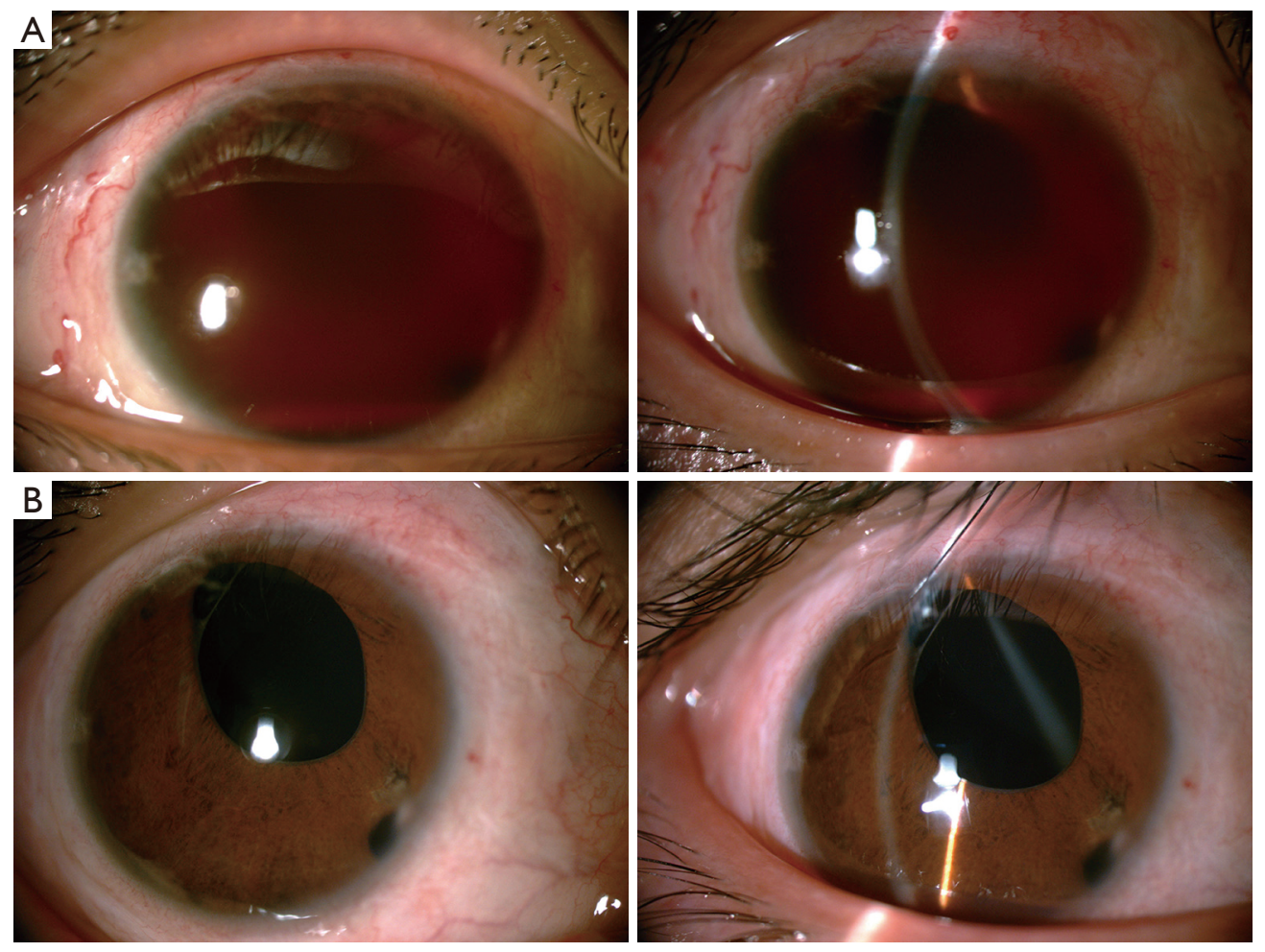

Figure 2 The participant with hyphema in the early stage after operation, which then absorbed itself. (A) One week after operation; (B) 6 months after operation.

(Figure $2 A, 2 B$ ). Up to the last follow-up, the FCVB was properly located in all patients, and was well distributed in the vitreous cavity (Figure $3 A, 3 B$ ) without silicone oil emulsification. None had postoperative complications like corneal vascularization, intraocular infection, or refractory ocular hypertension. Additionally, no FCVB rejection occurred in all cases, and no adverse complications such as conjunctival erosion, drain exposure (Figure $4 A, 4 B$ ), FCVB rupture, silicone oil spillage, or intolerable pain were recorded. There were 2 patients with marked corneal band-shaped degeneration before surgery, which was not significantly aggravated after surgery (Figure 5A-5C), and the other 3 patients had no significant change in corneal endothelial count before and after surgery.

In addition, up to the last follow-up, there was no significant change between preoperative and postoperative visual acuity. Before surgery, 4 patients had normal intraocular pressure while 1 patient had ocular hypotension; the latter was still at low level postoperatively, and the other patients had intraocular pressure in the normal range (Table 2). Postoperative UBM in the patient with ocular hypotension revealed ciliary body detachment, but no significant cleft.

\section{Discussion}

Silicone oil is an effective product that can treat diseases such as retinal detachment in a short period of time, but its prolonged retention in the eye causes a series of complications, such as silicone oil emulsification, replacement, and disruption of ciliary body function $(10,11)$. The eye becomes dependent on silicone oil, cannot be maintained, and ultimately requires enucleation. Therefore, materials that isolate the silicone oil are needed to degenerate and migrate the substitute into the ciliary body. The FCVB is different from the traditional supporting retinal mode, which supports the retina with a $360^{\circ}$ arc solid and has a long-lasting supporting effect on tears anywhere in the retina. By contrast with silicone oil tamponade, prone position is not required after FCVB (12). Intraocular pressure can also be adjusted by withdrawing silicone oil or injecting normal saline/silicone oil through 

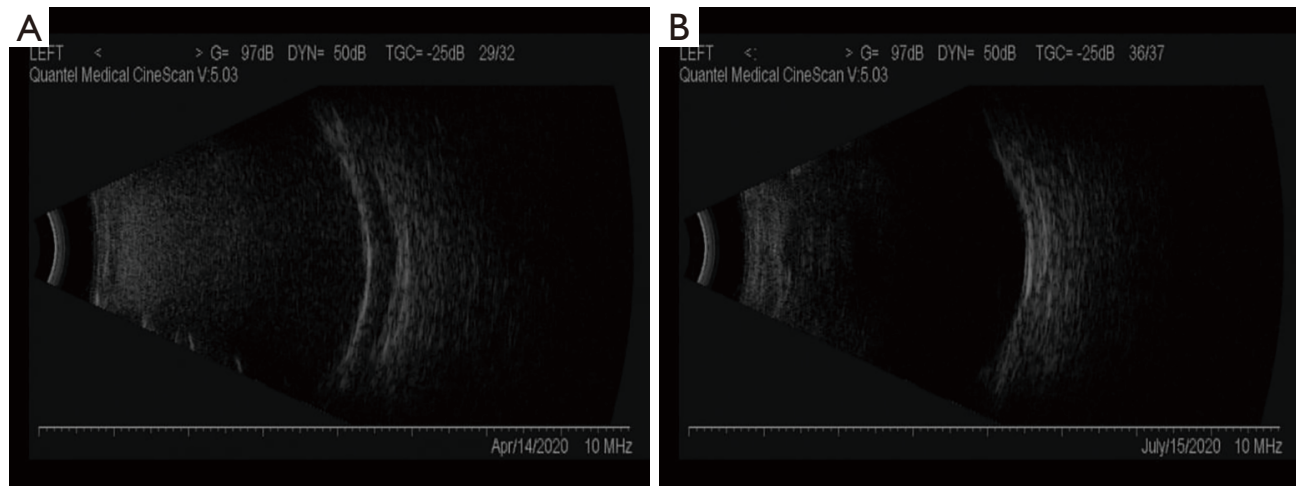

Figure 3 Comparison of ophthalmic B ultrasound examination. (A) B ultrasound before surgery; (B) B ultrasound at 3 months after surgery. G, gain; DYN, dynamic; TGC, time gain compensation.
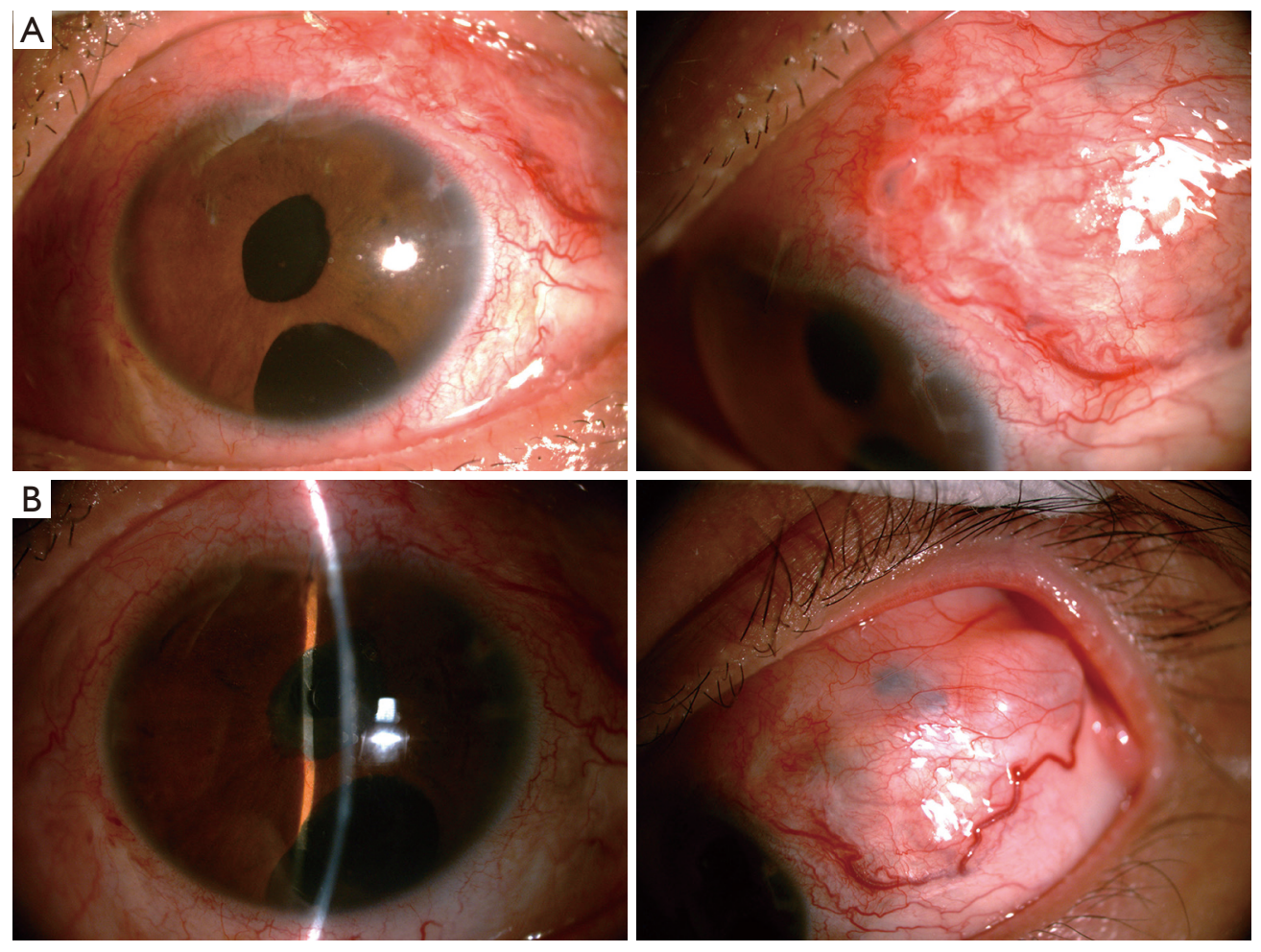

Figure 4 FCVB was well fixed without displacement, exposure, or rejection. (A) Two weeks after surgery; (B) 3 months after surgery. FCVB, foldable capsular vitreous body.

the drainage valve of FCVB (13). The changes of retinal blood vessels haven't been reported in FCVB implanted patients, one experimental study (14) suggested that FCVB did not gravely impact retinal vascular morphology or the retinal oxygenous microenvironment of intravitreal tamponade for 180 days in rabbits. This product is currently implanted in the eye for up to 10 years. In this study, 5 patients with silicone oil-dependent eyes received FCVB implantation, achieving the effects of restoring structure and basic function of the eyeball, supporting the retina, and maintaining good appearance. Our trial showed that FCVB is a safe and feasible artificial vitreous body with 

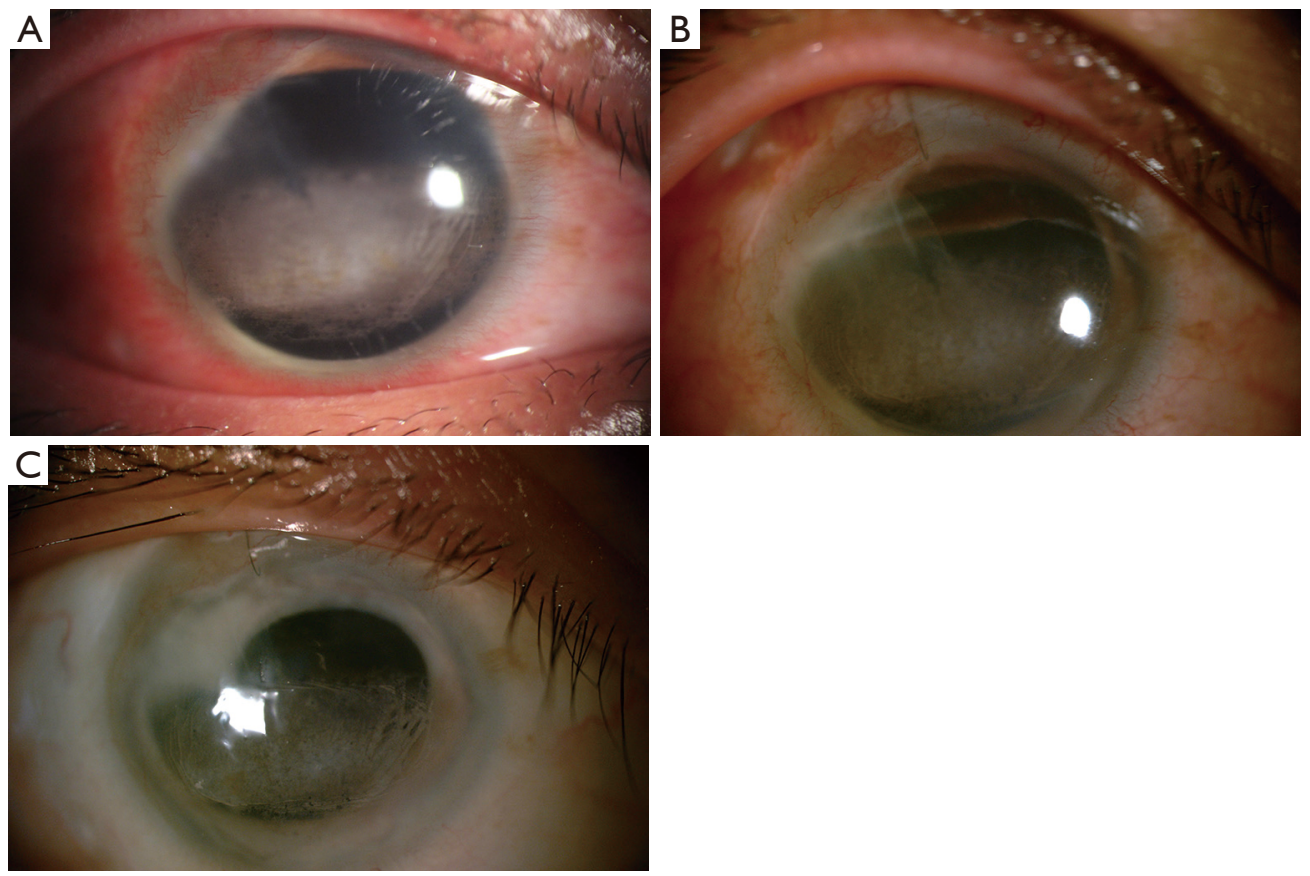

Figure 5 Corneal condition before and after operation. (A) Corneal conditions before operation; (B) 3 months after operation; (C) 12 months after operation.

Table 2 Comparison of visual acuity and intraocular pressure before and after FCVB

\begin{tabular}{|c|c|c|c|c|}
\hline Number & \multicolumn{2}{|c|}{ Before surgery } & \multicolumn{2}{|c|}{ Last follow-up } \\
\hline Patient 1 & Temporal light perception & Tn (corneal degeneration) & Temporal light perception & Tn (corneal degeneration) \\
\hline Patient 2 & Hand movement & 22 (NCT) & Hand movement & 11.1 (NCT) \\
\hline Patient 3 & Count finger & 17 (NCT) & Count finger & 14.6 (NCT) \\
\hline Patient 5 & Light perception & 10 (lcare) & Light perception & 11 (lcare) \\
\hline
\end{tabular}

FCVB, foldable capsular vitreous body; NCT, non-contact thermometer.

a significant clinical therapeutic effect for patients with silicone oil-dependent eyes.

The clinical application of FCVB is still in early stage with a large-scale promotion. Lin et al. (15) observed 11 patients with severe retinal detachment who were implanted with FCVB for 3 months; their results showed that FCVB could safely and effectively promote the anatomical retinal reattachment and could avoid complications such as silicone oil emulsification, silicone oil flowing into the anterior chamber, and corneal damage (16). The safety and efficacy of FCVB for severe ocular rupture and silicone oil- dependent eyes were also supported in the recent study by Zhang et al. (17) in 4 cases (4 eyes), and this product maintained ocular morphology well. In addition, in 2019, Zhang et al. (18) found that FCVB implantation for severe retinal detachment was safe and effective with good longterm stability through more than 1 year follow-up in 20 patients with severe trauma and silicone oil-dependent eyes. In our study, we hoped to know if consistent results could be obtained for FCVB implantation. During the observation, all patients had anatomical retinal reattachment, appropriate position of FCVB in the eye, good distribution of FCVB 
in the vitreous cavity, no silicone oil emulsification, and no severe irreversible complications. Only 1 case had postoperative hyphema, and the ocular condition improved and tended to be stable after treatment, showing no rebleeding. The results of our study further confirmed the clinical safety and efficacy of FCVB implantation.

In this study, 1 patient with ocular hypotension was analyzed. The level of intraocular pressure was still low after FCVB implantation, which may be caused by ciliary body detachment and poor function. We intend to assess ciliary body structure and function in further clinical studies, which may be the main cause of postoperative ocular hypotension. If preoperative UBM suggests that cyclodialysis cleft has been sutured intraoperatively, anti-inflammation needs to be strengthened postoperatively. However, FCVB implantation may not be able to relieve ocular hypotension in patients with poor ciliary body function, which is a concern for our future clinical study.

At present, silicone oil is commonly used as an artificial vitreous substitute, but often fails to maintain intraocular pressure for a long time; and its long-term filling will produce complications such as silicone oil emulsification, corneal degeneration, and secondary glaucoma $(14,19)$. Among them, the incidence of silicone oil emulsification is high. For example, Miller et al. (20) showed that silicone oil emulsification occurred in all 150 patients within 12 months. Another study further observed that silicone oil emulsification developed within 5-24 months, especially within 12 months (21). Therefore, the current clinical treatment for severe silicone oil-dependent eyes is more difficult. In this study, 5 participants were reexamined during the follow-up period of 12-15 months after operation. None of them had complications such as silicone oil emulsification, corneal degeneration/aggravated corneal degeneration, ocular hypertension, and all patients expressed satisfaction. The results of this study further confirm the advantages of FCVB implantation for silicone oil-dependent eyes.

There are some limitations in our study. First, there is a lack of detection data of postoperative axial length. Second, there was small sample size in this study. More cases and longer observation are still needed to further evaluate FCVB.

In conclusion, FCVB is a new potential treatment for severe silicone oil-dependent eyes, which can maintain ocular morphology and intraocular pressure without severe complications. The FCVB has been certificated by National Medical Products Administration in China and Confirmite
Europeenne (CE) the European Union, and applied for some patients clinically in Unite Kingdom, Belgium, Australia, Russia, Poland, Greece, Netherland etc. Recently, it was reported as the new material during scleral buckling surgery for the rhegmatogenous retinal detachment patients, who were simple, one-hole, and no proliferative vitreoretinopathy (22). Application makes the operation easier, more convenient, and painless. We will further expand the case collection and prolong the observation time for identify the clinical efficacy of FCVB providing more clinical experience.

\section{Acknowledgments}

Funding: This article was supported by Military Scientific Grant of Army Medical University (2020HQZX18).

\section{Footnote}

Reporting Checklist: The authors have completed the STROBE reporting checklist. Available at https://dx.doi. org/10.21037/apm-21-2554

Data Sharing Statement: Available at https://dx.doi. org/10.21037/apm-21-2554

Conflicts of Interest: All authors have completed the ICMJE uniform disclosure form (available at https://dx.doi. org/10.21037/apm-21-2554). The authors have no conflicts of interest to declare.

Ethical Statement: The authors are accountable for all aspects of the work in ensuring that questions related to the accuracy or integrity of any part of the work are appropriately investigated and resolved. This study was conducted in accordance with the Declaration of Helsinki (as revised in 2013) and was reviewed and approved by the Ethics Committee of the Southwest Hospital of Army Medical University (KY2021066). Informed consent was obtained from all the patients.

Open Access Statement: This is an Open Access article distributed in accordance with the Creative Commons Attribution-NonCommercial-NoDerivs 4.0 International License (CC BY-NC-ND 4.0), which permits the noncommercial replication and distribution of the article with the strict proviso that no changes or edits are made and the original work is properly cited (including links to both the 
formal publication through the relevant DOI and the license).

See: https://creativecommons.org/licenses/by-nc-nd/4.0/.

\section{References}

1. Mariacher S, Szurman P. Artificial vitreous body: Strategies for vitreous body substitutes. Ophthalmologe 2015;112:572-9.

2. Gao QY, Fu Y, Hui YN. Vitreous substitutes: challenges and directions. Int J Ophthalmol 2015;8:437-40.

3. Barth H, Crafoord S, Andréasson S, et al. A cross-linked hyaluronic acid hydrogel (Healaflow(®)) as a novel vitreous substitute. Graefes Arch Clin Exp Ophthalmol 2016;254:697-703.

4. Zhang R, Wang T, Xie C, et al. Evaluation of supporting role of a foldable capsular vitreous body with magnetic resonance imaging in the treatment of severe retinal detachment in human eyes. Eye (Lond) 2011;25:794-802 .

5. Gao Q, Mou S, Ge J, et al. A new strategy to replace the natural vitreous by a novel capsular artificial vitreous body with pressure-control valve. Eye (Lond) 2008;22:461-8.

6. Wang P, Gao Q, Jiang Z, et al. Biocompatibility and retinal support of a foldable capsular vitreous body injected with saline or silicone oil implanted in rabbit eyes. Clin Exp Ophthalmol 2012;40:e67-75.

7. Liu Y, Ke Q, Chen J, et al. Sustained mechanical release of dexamethasone sodium phosphate from a foldable capsular vitreous body. Invest Ophthalmol Vis Sci 2010;51:1636-42.

8. Chen H, Feng S, Liu Y, et al. Functional evaluation of a novel vitreous substitute using polyethylene glycol sols injected into a foldable capsular vitreous body. J Biomed Mater Res A 2013;101:2538-47.

9. Zeng B, Wang Q, Sui G, et al. Foldable capsular vitreous body implantation for treatment of traumatic retinal detachment: two case reports. J Int Med Res 2021;49:300060521990257.

10. Zafar S, Bokhari SA, Kamil Z, et al. Outcomes of silicone oil removal. J Coll Physicians Surg Pak 2013;23:476-9.

11. Khoroshilova-Maslova IP, Nabieva MK, Leparskaia NL. Morphogenesis of complications after long-term

Cite this article as: Liu N, Kang L, Yu X, Lv Q, Xi L, Liu M, Liu Y, Wu N. Preliminary clinical application of foldable capsular vitreous body in severe silicone oil-dependent eyes. Ann Palliat Med 2021;10(10):10922-10929. doi: 10.21037/apm-212554 intraocular silicon oil filling (clinical histopathological study). Vestn Oftalmol 2012;128:57-61.

12. Ye YH, Tan SF, Wu SH. Care of artificial vitreous implanted in the treatment of patients with severe ocular trauma retinal detachment. Modern Hospital 2013;13:75-6.

13. Gao Q, Chen X, Ge J, et al. Refractive shifts in four selected artificial vitreous substitutes based on GullstrandEmsley and Liou-Brennan schematic eyes. Invest Ophthalmol Vis Sci 2009;50:3529-34.

14. Yang W, Yuan Y, Zong Y, et al. Preliminary study on retinal vascular and oxygen-related changes after longterm silicone oil and foldable capsular vitreous body tamponade. Sci Rep 2014;4:5272.

15. Lin X, Ge J, Gao Q, et al. Evaluation of the flexibility, efficacy, and safety of a foldable capsular vitreous body in the treatment of severe retinal detachment. Invest Ophthalmol Vis Sci 2011;52:374-81.

16. Lin $X$, Sun $X$, Wang Z, et al. Three-year efficacy and safety of a silicone oil-filled foldable-capsular-vitreousbody in three cases of severe retinal detachment. Transl Vis Sci Technol 2016;5:2.

17. Zhang G, Gong H, Hui Y, et al. Preliminary clinical practice in implantation of foldable capsular vitreous body. International Eye Science 2018;18:578-80.

18. Zhang X, Tian X, Zhang B, et al. Study on the effectiveness and safety of Foldable Capsular Vitreous Body implantation. BMC Ophthalmol 2019;19:260.

19. Lin $X$, Wang $Z$, Jiang $Z$, et al. Preliminary efficacy and safety of a silicone oil-filled foldable capsular vitreous body in the treatment of severe retinal detachment. Retina 2012;32:729-41.

20. Miller JB, Papakostas TD, Vavvas DG. Complications of emulsified silicone oil after retinal detachment repair. Semin Ophthalmol 2014;29:312-8.

21. Toklu Y, Cakmak HB, Ergun SB, et al. Time course of silicone oil emulsification. Retina 2012;32:2039-44.

22. Zhang B, Li C, Jia Y, et al. A pilot clinical study of treating rhegmatogenous retinal detachment by silicone rubber balloon scleral buckling. Retina 2020;40:1918-28.

(English Language Editor: J. Jones) 